\title{
Adenotonsillectomy improves slow-wave activity in children with obstructive sleep
} apnoea

\author{
N. Ben-Israel*, Y. Zigel ${ }^{*}$, A. Tal*, ${ }^{*}$, Y. Segev ${ }^{+}$and A. Tarasiuk*
}

ABSTRACT: The aim of the present study was to estimate slow-wave activity (SWA), a marker of sleep homeostasis, in children with obstructive sleep apnoea (OSA) before and after adenotonsillectomy (AT) compared with untreated OSA children (comparison group).

14 children with OSA (mean \pm SD age $6.4 \pm 2.5 \mathrm{yrs}$; apnoea-hypopnoea index (AHI) $10.0 \pm 10.3$ events $\cdot h^{-1}$ ) who underwent AT were consecutively recruited to the study. The comparison group comprised six retrospectively recruited children (age $5.4 \pm 2.2$ yrs; AHI $9.4 \pm 7.6$ events $\cdot h^{-1}$ ) with OSA that did not undergo treatment. Electroencephalogram (derivation C3/A2) was analysed using spectral and waveform analysis to determine SWA energy and slowwave slope. The same procedure was repeated 5.4 and 19 months later for the AT and comparison groups, respectively.

AT improved respiration without a change in duration of sleep stages. Following AT, $>50 \%$ elevation of SWA during the first two sleep cycles $(p<0.01)$ and a more physiological decay of SWA across the night $(p<0.0001)$ were noted. The slow-wave slope increased by $>30 \%$ following AT $(p<0.03)$. No significant changes were found in SWA in the comparison group.

Sleep homeostasis is considerably impaired in pre-pubescent children with OSA. AT restores more physiological sleep homeostasis in children with OSA. SWA analysis may provide a useful addition to standard sleep-stage analyses in children with OSA.

KEYWORDS: Adenotonsillectomy, children, obstructive sleep apnoea, slow-wave activity

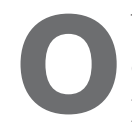
bstructive sleep apnoea (OSA) is a common condition with an estimated prevalence of $1-3 \%$. The most common cause of OSA in children is adenotonsillar hypertrophy; thus, adenotonsillectomy (AT) is the treatment of choice. Following AT, the majority of children with OSA will have improvement in neurobehavioural function and growth [1-4], and a decrease in healthcare utilisation. [5]

Although paediatric OSA causes substantial neurobehavioural morbidity, at least in part through sleep fragmentation, previous comparative polysomnography (PSG) data have revealed no consistent PSG differences following AT $[1,4,6,7]$. Some studies demonstrated improvement in sleep efficiency, decreased stage 1 sleep and increased slow-wave sleep (SWS) [2, 8, 9]. Studies exploring distributions of contiguous sleep duration following AT also found no consistent findings. TAL et al. [1] reported no changes in cumulative median duration of sleep stages after AT, while others found some changes, mainly in stage 2 [10]. The cumulative data on the effect of AT on sleep stages using traditional PSG scoring are too small to carry the obvious consistent improvement in clinical, neurocognitive, growth and endocrine changes following AT in children with OSA.

Spectral analysis makes possible the quantitative description of the timecourse of sleep electroencephalogram (EEG) across the night. Spectral analysis of slow-wave activity (SWA) is a quantitative measure of slow-wave sleep dynamics, and represents a physiological marker of homeostatic regulation of sleep [11]. Stronger cortical connections would produce stronger network synchronisation and, thus, a higher level of SWA, whereas weaker connections would reduce network synchronisation and, thereby, SWA $[12,13]$. The magnitude of the slope of slow waves can represent an additional measure of SWA [14]. To the best of our knowledge, no studies have been performed on SWA in OSA children before and after AT. In adult OSA patients, HEINZER et al. [15] reported a significant increase in mean SWA after

\section{AFFILIATIONS}

*Sleep-Wake Disorders Unit, Soroka University Medical Center and Dept of Physiology,

+Shraga Segal Dept of Microbiology and Immunology, Faculty of Health Sciences,

\#Dept of Biomedical Engineering, Faculty of Engineering, and -Dept of Paediatrics, Ben-Gurion University of the Negev, Beer-Sheva, Israel.

CORRESPONDENCE

A. Tarasiuk

Dept of Physiology, Faculty of Health Sciences

Ben-Gurion University of the Negev P0 box 105

Beer-Sheva 84105

Israel

E-mail: Tarasiuk@bgu.ac.il

Received:

July 092010

Accepted after revision: Aug 192010

First published online:

Sept 032010 
continuous positive airway pressure (CPAP) and restored a physiological decay of SWA across the night.

In the present study, we explored SWA in children with OSA before and after AT compared with untreated OSA children (comparison group). We hypothesise that SWA will increase following AT in children with OSA.

\section{METHODS}

\section{Patients}

Consecutive, otherwise healthy children referred to the SleepWake Disorder Unit, Soroka University Medical Center, BeerSheva, Israel for evaluation of possible OSA were included in the study. Children with other chronic medical illnesses, genetic disorders, facial anomalies and two subjects studies with poor quality EEG signals (one from each group) were excluded. Two groups of children were investigated: the treated group $(n=14)$ was consecutively recruited and included children with PSG-proven OSA undergoing AT. The comparison group $(n=6)$ were retrospectively recruited; these were children with PSG-proven OSA who did not undergo surgery or CPAP treatment (table 1). The institutional ethics committee approved the study protocol.

\begin{tabular}{|c|c|c|c|c|}
\hline \multirow{2}{*}{ BLE } & \multicolumn{2}{|c|}{ AT } & \multicolumn{2}{|c|}{ Comparison } \\
\hline & 1st PSG & 2nd PSG & 1st PSG & 2nd PSG \\
\hline Subjects $\mathbf{n}$ & \multicolumn{2}{|c|}{14} & \multicolumn{2}{|c|}{6} \\
\hline Age yrs & $6.4 \pm 2.5$ & $7.3 \pm 2.7$ & $5.4 \pm 2.2$ & $7 \pm 2.9$ \\
\hline Males/females $n$ & \multicolumn{2}{|c|}{$7 / 7$} & \multicolumn{2}{|c|}{$4 / 2$} \\
\hline Lights-off time $h$ & $21: 32 \pm 00: 33$ & $21: 19 \pm 00: 22$ & \multicolumn{2}{|c|}{$21: 43 \pm: 00: 06$ 21:12 $\pm 00: 25^{\star}$} \\
\hline TST min & $424.5 \pm 22.7$ & $432.9 \pm 34.9$ & $393.5 \pm 54.2$ & $448 \pm 24.9^{*}$ \\
\hline Sleep efficiency \% & $84.3 \pm 10.1$ & $83.6 \pm 10.5$ & $83.5 \pm 7.1$ & $79.2 \pm 7.5^{\star}$ \\
\hline WASO min & $24.8 \pm 17.1$ & $31.2 \pm 30$ & $27.8 \pm 12.1$ & $59.7 \pm 26.8^{*}$ \\
\hline $\begin{array}{c}A r+A w \text { index } \\
\text { events } \cdot h^{-1}\end{array}$ & $19.8 \pm 10.2$ & $14.0 \pm 7.3^{\#}$ & $16.0 \pm 6.0$ & $21.2 \pm 6.6^{\#}$ \\
\hline S1 \% & $1.7 \pm 1.6$ & $1.3 \pm 2.1$ & $0.3 \pm 0.5$ & $1.5 \pm 1.8$ \\
\hline S2 \% & $49.9 \pm 7.5$ & $48.9 \pm 7.1$ & $56.5 \pm 9.3$ & $59.2 \pm 7.5$ \\
\hline s3+4 \% & $30.6 \pm 7.4$ & $33.7 \pm 6.3$ & $29.3 \pm 1.0$ & $26.7 \pm 6.3$ \\
\hline REM \% & $16.0 \pm 7.2$ & $15.0 \pm 8.6$ & $13.8 \pm 8.8$ & $12.6 \pm 7.0$ \\
\hline \multicolumn{5}{|l|}{$A H I$ events $\cdot h^{-1}$} \\
\hline Total & $10.0 \pm 10.3$ & $1.1 \pm 1.0^{\#}$ & $9.4 \pm 7.6$ & $13.1 \pm 7.7$ \\
\hline NREM & $9.8 \pm 11$ & $0.8 \pm 1.4$ & $8.8 \pm 8.2$ & $15.6 \pm 9$ \\
\hline REM & $15.1 \pm 16.7$ & $5.0 \pm 10.3$ & $19.3 \pm 10$ & $23.5 \pm 22.6$ \\
\hline \multicolumn{5}{|l|}{$S_{p}, \mathrm{O}_{2} \%$} \\
\hline Mean waking & $96.1 \pm 1.0$ & $97.5 \pm 1.2^{\#}$ & $97.6 \pm 1.0$ & $96 \pm 2.1$ \\
\hline Nadir & $86.1 \pm 10.2$ & $92.9 \pm 1.7^{\#}$ & $90.7 \pm 8.1$ & $90.5 \pm 3.6$ \\
\hline
\end{tabular}

Data are presented as mean $\pm \mathrm{SD}$, unless otherwise stated. PSG: polysomnography; TST: total sleep time; WASO: wake after sleep onset; Ar: arousal; AW: awakening; S1: sleep stage 1; S2: sleep stage 2; S3+4: sleep stages 3 and 4; REM: rapid eye movement sleep; AHI: apnoea-hypopnoea index; NREM: nonREM sleep; $\mathrm{Sp}_{2} \mathrm{O}_{2}$ : arterial oxygen saturation measured by pulse oximetry. *: $p<0.02 ;{ }^{*}: p<0.05$.
The diagnostic evaluation included a detailed clinical history obtained using a validated questionnaire in Hebrew [3] that is based on previous questionnaires $[2,3,16]$. Physical examination was performed by a paediatric pulmonologist and an otolaryngologist.

\section{PSG study}

In the laboratory, data were acquired using a computerised, commercially available sleep-monitoring system (SomnoStar Pro; Viasys, Yorba Linda, CA, USA) as described previously [1]. Parents were instructed to maintain their children's normal daily sleep-wake routine and to avoid consumption of soft drinks on the day of the study. Overnight PSG monitoring included recordings of EEG (referential derivations, international 10-20 system: C3/A2, C4/A1, and O2/A1, O1/A2), electrooculogram (right and left outer canthus), electromyogram and ECG. Derivation C3/A2 was used for sleep-stage scoring and for power spectral analysis; similar results were obtained using C4/A1. Airflow was recorded using a pressure transducer (Pro Tech Monitoring Inc., Odessa, FL, USA), chest and abdominal efforts were recorded using inductive plethysmography (RespiTrace Plus; Viasys) and haemoglobin oxygen saturation was monitored by pulse oximetry (Philips Respironics, Pittsburgh, PA, USA). Scoring was performed by a trained technician and reviewed by a trained polysomnographer. Sleep-wake and sleep stages were scored according to the criteria of RECHTSCHAFFEN and KALES [17]. Non-rapid eye movement (NREM) sleep episodes were defined according to standard criteria [18], and adjusted for children [19] because of the frequent occurrence of a "skipped" rapid eye movement (REM) sleep episode after the first NREM sleep episode. Arousals and awakenings were scored using American Sleep Disorders Association criteria [20] with appropriate modifications for children [1]. Obstructive respiratory events during sleep were scored using the traditional criteria [21]. Obstructive apnoea was defined as paradoxical breathing for at least two respiratory cycles with complete cessation of nasal airflow. A hypopnoea was scored when the paradoxical breathing was accompanied by a reduction of $\geqslant 50 \%$ in airflow, resulting in either an arousal or in oxygen desaturation of $\geqslant 4 \%$. Apnoeahypopnoea index (AHI) was calculated as the number of respiratory events per hour of sleep.

\section{EEG spectral and waveform analysis}

The EEG signals (C3/A2 and C4/A1) were sampled at $100 \mathrm{~Hz}$ (12-bit AD converter) and band-pass filtered $(0.3-35 \mathrm{~Hz})$. Epochs (30-s segments) that contained artefacts, such as movement and ocular or muscle artefacts, were removed. Respiratory-induced artefacts were removed; these artefacts were minimal when the standard EEG arousal criteria were employed $[1,22]$. As a consequence, an average of 4.3 and $5.2 \%$ of the epochs were excluded from the first and the second study night, respectively. The EEG was analysed off-line (MATLAB R-2008a; The MathWorks, Inc., Natick, MA, USA).

Power spectrum was estimated by fast Fourier transform. Each 30-s epoch was subdivided into 4-s (400-sample) subsegments (2-s subsegments yielded similar results) with a 2-s overlap using a hamming window (14 segments in each epoch); the power spectral density was averaged, resulting in a $0.25-\mathrm{Hz}$ bin. The absolute energy in each frequency band, expressed in 
squared microvolts, was calculated for the following bands: $\delta$ frequency (often referred to as SWA; 0.75-4 Hz); $\theta$ frequency (4-8 Hz); $\alpha$ frequency $(8-12 \mathrm{~Hz}) ;$ and $\beta$ frequency $(12-16 \mathrm{~Hz})$. The total energy of each frequency band was calculated for every 30-s epoch. A smoothed version of the $\delta$ energy curve (12-epoch moving average) allows more accurate extraction of the maximum, minimum and mean of $\delta$ energy in every sleep cycle. For standardisation of EEG signal analysis, amplitudes were normalised using two approaches: first, to total energy of an artefact-free all-night EEG signal and secondly, by the mean energy of sleep stage 2 alone.

Waveform analysis was performed according to RIEDER et al. [23]. The slow-wave slope was defined as the amplitude of the most negative peak divided by the time to the next zero crossing of a band-pass filtered $(0.75-4 \mathrm{~Hz})$ and smoothed version of the EEG signal. Only waves with consecutive zero crossing separated by $0.25-1$ s were considered slow waves. The calculated slow-wave slopes were averaged in every 30-s epoch. Both ascending and descending wave slopes revealed similar behaviour and, therefore, only the first was examined.

\section{Data analysis}

Statistical analysis was performed using MATLAB. Data are presented as mean \pm SD unless otherwise specified. PSG data were compared using two-tailed t-tests for paired groups. A linear regression test was performed to determine the correlation between SWA and the slope, and between SWA and AHI or arterial oxygen saturation measured by pulse oximetry $\left(\mathrm{Sp}, \mathrm{O}_{2}\right)$. Two-way ANOVA (ANOVA-2) was used to compare SWA (or slow-wave slope) before and after AT or no treatment (factor 1) and sleep duration (factor 2). The null hypothesis was rejected at the $5 \%$ level.

\section{RESULTS}

\section{Patients}

20 children participated in this study. Daily routine at home was lights-out between 21:00 and 22:00 h, and mean sleep duration was $9.3 \mathrm{~h}$ (range $8-10 \mathrm{~h}$ ), with no significant difference between groups. Mean \pm SD ages of the AT and comparison groups at the first PSG were $6.4 \pm 2.5$ and $5.4 \pm 2.3 \mathrm{yrs}$, respectively, and at the second PSG study, mean ages were $7.3 \pm 2.7$ and $7 \pm 2.9$ yrs, respectively. All subjects were in the pre-puberty age range. The time between the first and second PSG studies was 10.5 \pm 7 (range 4-25) and 19 \pm 20 (range 662) months for the treated and untreated group, respectively $(\mathrm{p}=0.34)$. For the treated group, post-PSG studies were repeated $5.4 \pm 4$ (range 1.5-18) months after the date of surgery. The symptoms reported by the parents for all 20 children prior to the first PSG study are summarised in table 2. Parents reported mouth breathing during wakefulness, restless sleep, habitual loud snoring, behavioural problems and upper airway infections. Most parents (12 out of 20 parents) were quite concern about their children's symptoms, which motivated them to seek medical help.

\section{Respiratory characteristics}

All respiratory parameters improved significantly after AT (table 1). Mean \pm SD AHI decreased from $10.0 \pm 10.3$ to $1.1 \pm$ 1.0 events $\cdot h^{-1}(p<0.002)$ after AT surgery. Following AT, five out of 14 children had REM AHI equal to NREM AHI, while the rest exhibited higher REM AHI. Nadir $\mathrm{Sp}_{\mathrm{p}} \mathrm{O}_{2}$ significantly

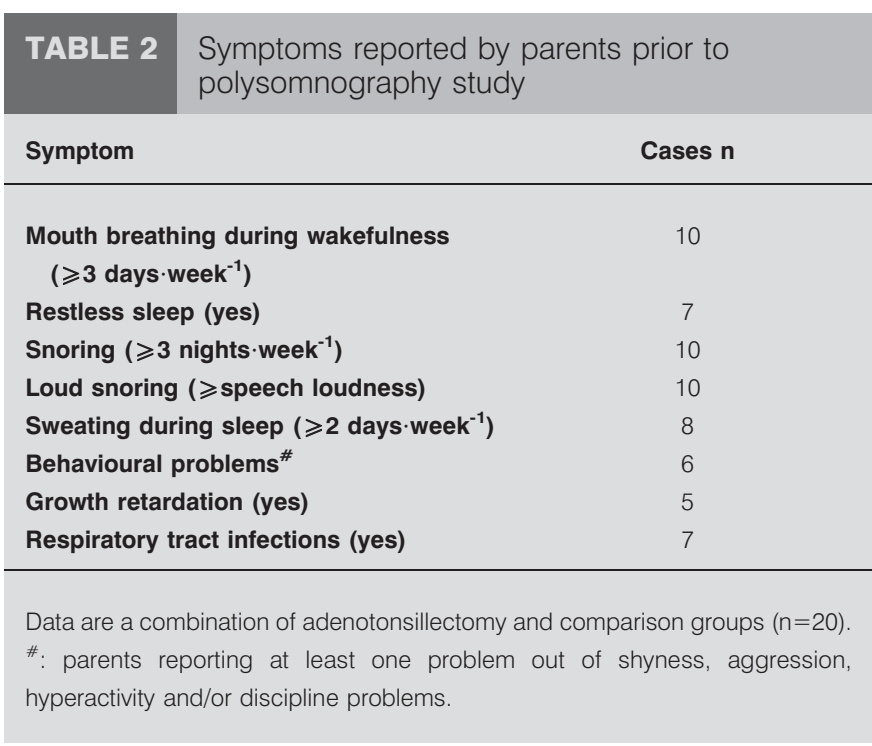

improved after AT surgery (table 1). In the comparison group, a trend toward increased AHI was observed when comparing the first PSG results to second PSG results, from $9.4 \pm 7.6$ to $13.1 \pm 7.7$ events $\cdot h^{-1}(p=0.07)$, respectively.

\section{Sleep architecture}

In the AT group, there were no significant changes in the cumulative duration spent in any of the sleep stages and sleep efficiency (table 1). Arousal plus awakening (Ar+Aw) index was significantly decreased following AT (table 1). Most of the arousals were spontaneously induced and $<15 \%$ were respiratory-induced. In the nontreated group, there were no significant changes in the cumulative duration spent in the sleep stages and sleep efficiency. Ar+Aw index increased in the second PSG study $(\mathrm{p}=0.04$; table 1$)$.

\section{EEG spectral and waveform analysis}

For both groups, the total energy of sleep stage 2 was $15.1 \pm 5.4$ and $15.1 \pm 2.7 \mu \mathrm{V}^{2}$ during the first and second PSG studies $(p=0.6)$, respectively, without significant differences between the two groups $(p=0.57)$. Therefore, signals were normalised to whole-night total energy and mean energy of sleep stage 2 . Both normalisation methods yielded similar results; therefore, in this study, the presented data correspond to the normalisation of sleep stage 2 energy. The normalised power values of the $\theta, \alpha$ and $\beta$ frequency bands did not change significantly during the night between the first and second PSG studies (treatment factor $F=0.53(p<0.47), F=0.01 \quad(p<0.93)$ and $F=0.03(p<0.87)$, respectively, by ANOVA-2; data not shown). Similar results were obtained for the comparison group. The all-night EEG power spectra for SWS (stages 3 and 4) and REM (treated group), before and after AT, are illustrated in figure 1. Normalised EEG power densities during SWS significantly improved following AT in the $\delta$ frequency range $(0.75-4 \mathrm{~Hz}$; treatment factor $\mathrm{F}=39.4$ $(\mathrm{p}<0.0001)$ by ANOVA-2), with no significant change in other frequency bands, i.e. $\theta, \alpha$ and $\beta$. No significant changes were noted in the power frequency spectrum of REM sleep stage (treatment factor $\mathrm{F}=0.79, \mathrm{p}<0.37$ ). For the comparison group, no significant changes were noted in power spectra for both SWS or REM (data not shown). 

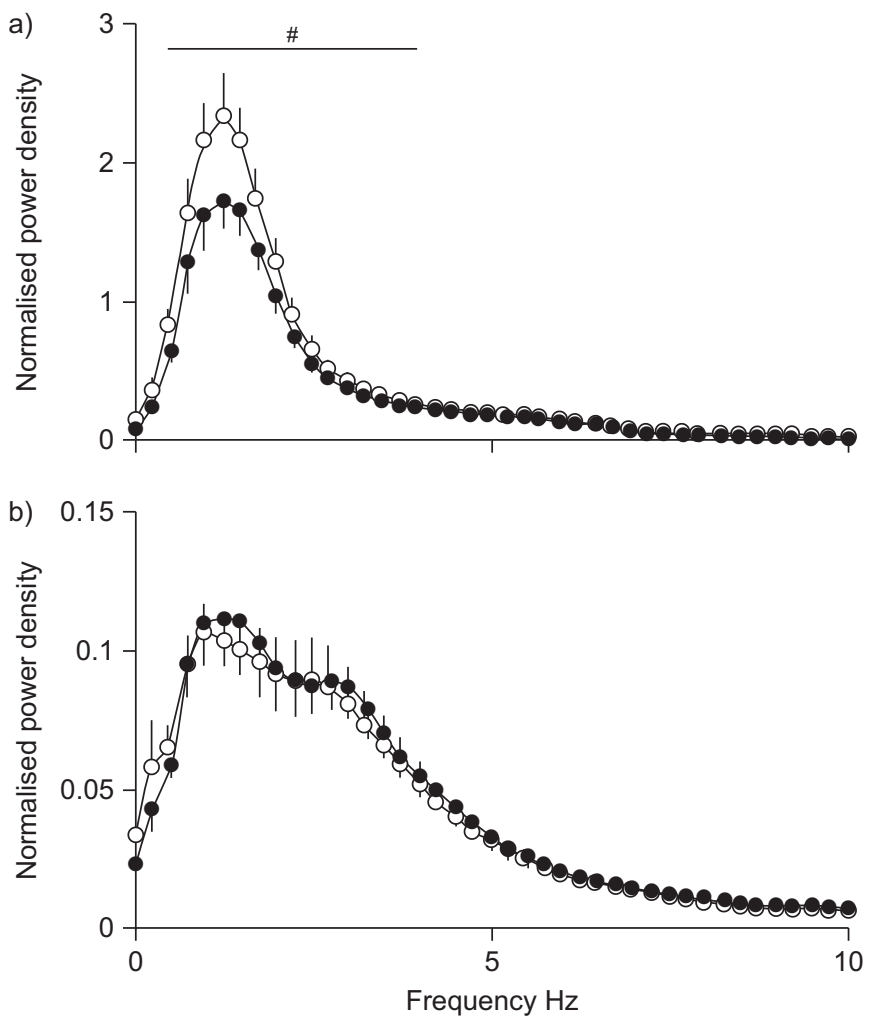

FIGURE 1. Mean \pm SEM all-night electroencephalogram normalised power spectral density for a) non-rapid eye movement short-wave sleep (stages 3-4) and b) rapid eye movement sleep before $(\bullet)$ and after adenotonsillectomy $(\bigcirc)$. Power density values (normalised) are plotted every $0.25-\mathrm{Hz}$ bin. For the purpose of clarity only, the frequency range of $0-10 \mathrm{~Hz}$ is shown. ${ }^{*}$ : treatment factor $\mathrm{F}=39.4$, $p<0.0001$.

A close association was found between SWA and slope: the greater the energy, the steeper the slope for both groups and both PSG studies (e.g. fig. 2c, d, g and h). The timecourse of the slow-wave slope across four sleep cycles is demonstrated in figure 3 . The treated group showed a significant increase in the slope of slow waves following AT across the first three sleep cycles (treatment factor $\mathrm{F}=4.7(\mathrm{p}<0.025)$ by ANOVA-2), while SWA increased significantly only in the first two cycles (fig. 4).

The nocturnal timecourse of sleep stages (fig. 2a and b), absolute SWA (fig. 2c and d), smoothed SWA curve (fig. 2e and $\mathrm{f}$ ) and slow-wave slope (fig. $2 \mathrm{~g}$ and $\mathrm{h}$ ) are illustrated in figure 2 for a single participant before (fig. 2a, c, e and g) and after (fig. 2b, d, f and h) AT. No significant change was found in any of the sleep-stage durations (fig. $2 a$ and $b$, and table 1). However, AT induced considerable elevation of $\delta$ energy (i.e. SWA; fig. 2c-f) and in the slow-wave slope (fig. $2 \mathrm{~g}$ and $\mathrm{h}$ ). The mean maximal $\delta$ energy across the first four sleep cycles in the AT group are summarised in figure $4 \mathrm{a}$ and comparison group in figure $4 \mathrm{~b}$. At the first PSG study, both groups had a statistically similar SWA pattern (group factor $\mathrm{F}=0.19$, $\mathrm{p}<0.662$; sleep cycle factor $\mathrm{F}=3.73, \mathrm{p}<0.016$; ANOVA-2).

Following AT, a considerable elevation ( $>50 \%$ ) of maximal SWA during the first two sleep cycles was noted (treatment factor $\mathrm{F}=6.9, \mathrm{p}<0.01$; ANOVA-2). Moreover, AT led to a more physiological decay of SWA during the night (fig. 4a; sleep cycle factor $\mathrm{F}=20.35, \mathrm{p}<0.0001$; ANOVA-2). No significant interaction was found between $\delta$ energy and sleep cycle $(\mathrm{F}=1.07, \mathrm{p}=0.37 ;$ ANOVA-2). No significant changes were noted in normalised SWA energy of the comparison group (fig. $4 \mathrm{~b}$; treatment factor $\mathrm{F}=0.06, \mathrm{p}=0.8$; ANOVA-2).

\section{DISCUSSION}

In pre-pubescent children with OSA, AT improved sleep depth, without any significant change in the cumulative proportion of sleep stage duration. SWA analysis may provide useful additions to the standard sleep-stage analyses.

\section{Subjects}

We included prospectively recruited, otherwise healthy children with typical symptoms for OSA. Removal of the adenotonsillar tissue resulted in improvement of respiration during sleep, but it is possible that children may not have been completely treated by AT, as indicated by abnormal drops of $\mathrm{Sp}_{\mathrm{p}} \mathrm{O}_{2}$ to $93 \%$ and REM-related AHI. Our findings confirm previous reports demonstrating that residual OSA is present in a large proportion of children after AT, mainly in REM sleep [1, $7,8]$. SWA will not change much if the residual obstructive events following AT occurred during REM sleep, since it is a phenomenon related to NREM sleep. The comparison group retrospectively recruited included otherwise healthy children with OSA who did not adhere to the recommended therapy. In the first PSG study, the comparison group was statistically similar to the AT group in OSA severity parameters. The lack of adherence to the recommended therapy was mainly due to the low level of awareness and doubts among parents, physicians and administrators of the benefits of early AT intervention.

Changes observed in SWA following AT probably reflect a "true" change in sleep depth over treatment. All included subjects in our study were children that, at the conclusion of the second PSG study, were in their pre-puberty age range; therefore, changes in SWA are unlikely to be related to puberty. SWA undergoes dramatic changes during brain maturation; it maintains stability across childhood and declines sharply across puberty [24]. A possible limitation of the present study is the "first-night effect", which may affect the sleep architecture of individuals during their first night of the PSG evaluation. However, in a previous study we have shown that the only significant change between two consecutive nocturnal PSG studies was in REM sleep [25]. No change was noted in SWA in the comparison group between the two PSG studies, where each patient served as their own control; thus, we do not think the first-night effect affects our interpretations.

\section{SWA}

EEG signals were normalised to stage 2 energy for standardisation between subjects; EEG normalisation to the whole-night energy yields similar SWA trends and magnitudes. In the present study, normalised SWA improved considerably by $>50 \%$ during the first two sleep cycles following AT compared to the comparison group, supporting the hypothesis that sleep depth is enhanced following surgery. Our findings support the idea that sleep-disordered breathing may prevent physiological homeostatic sleep-drive dissipation, which critically depends on uninterrupted sleep. Others have shown that SWA significantly decreased among adult OSA patients compared to normal controls when artefact-free EEG signals 

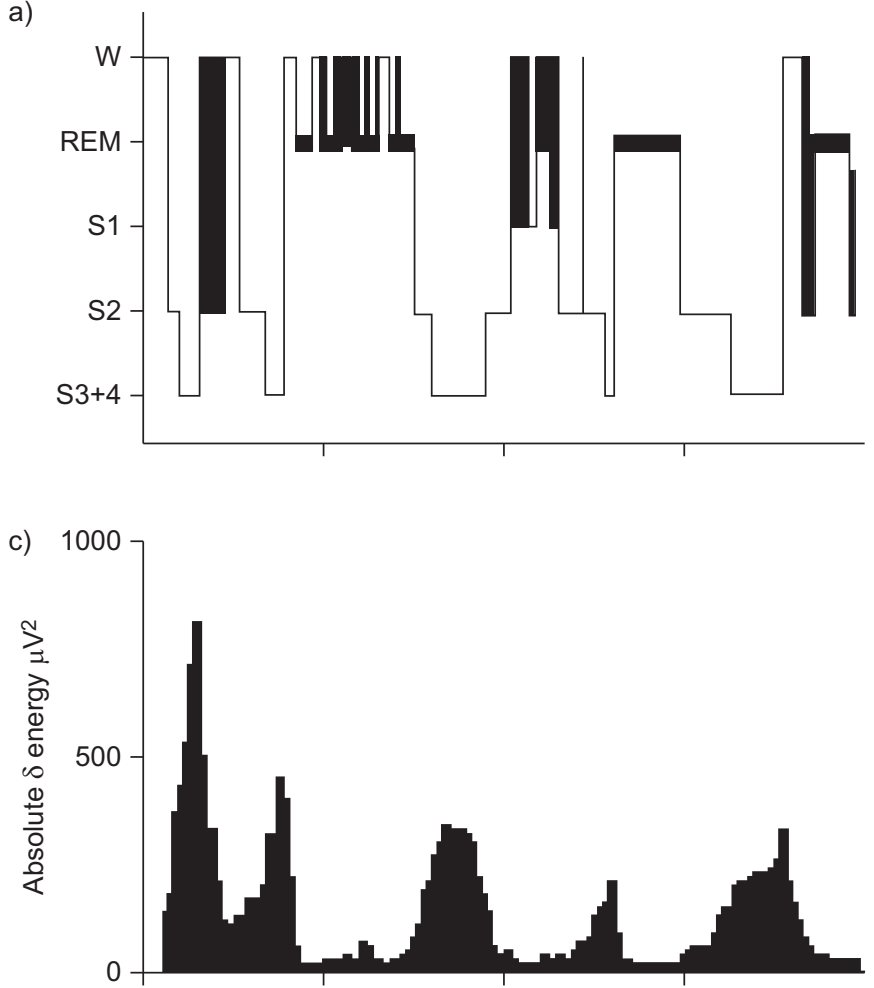

e)
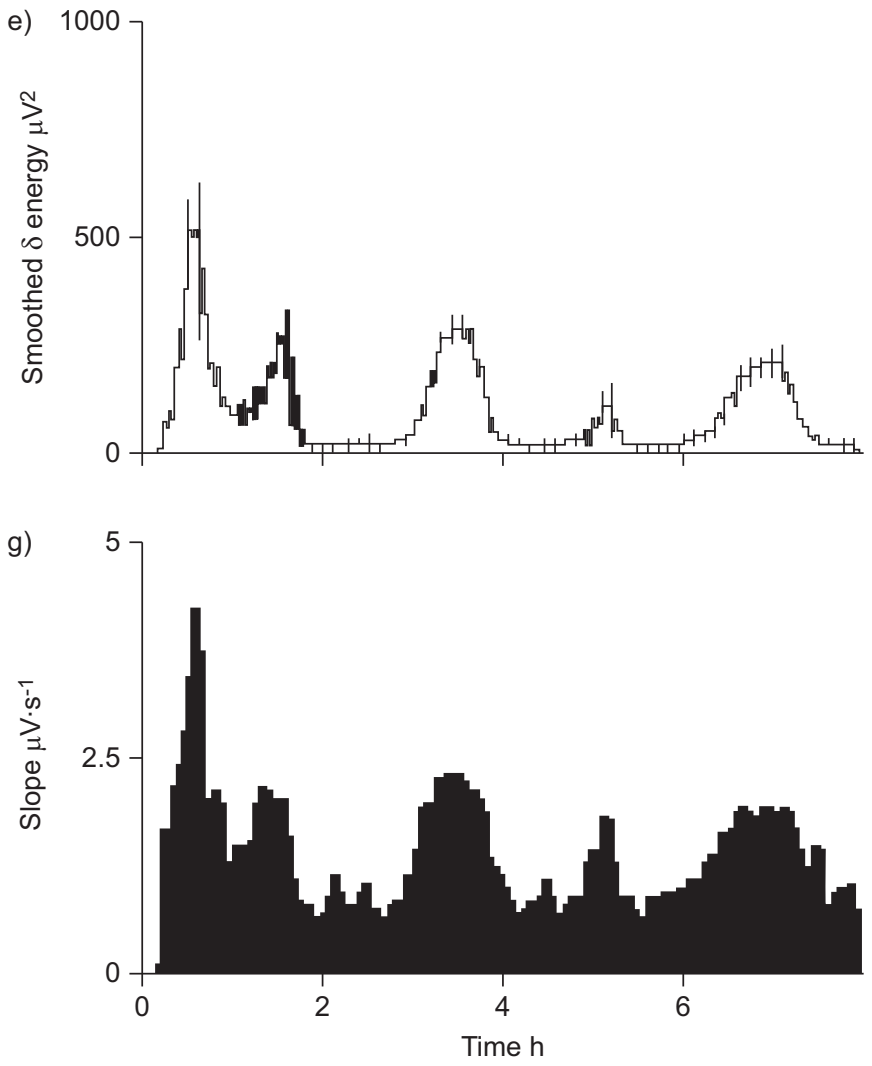

b)

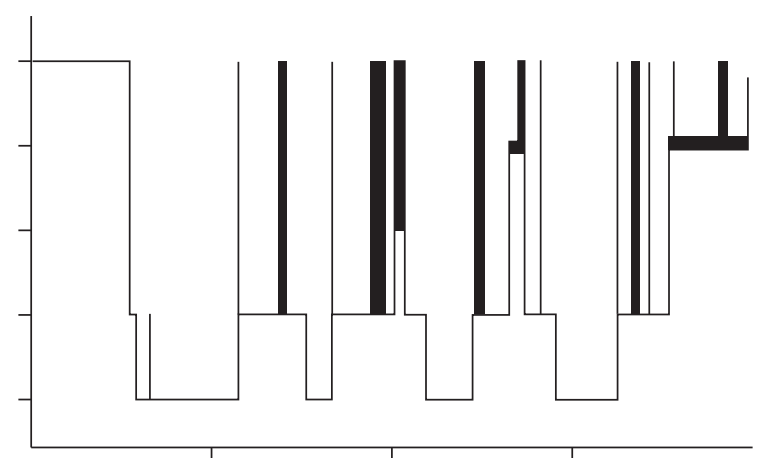

d)

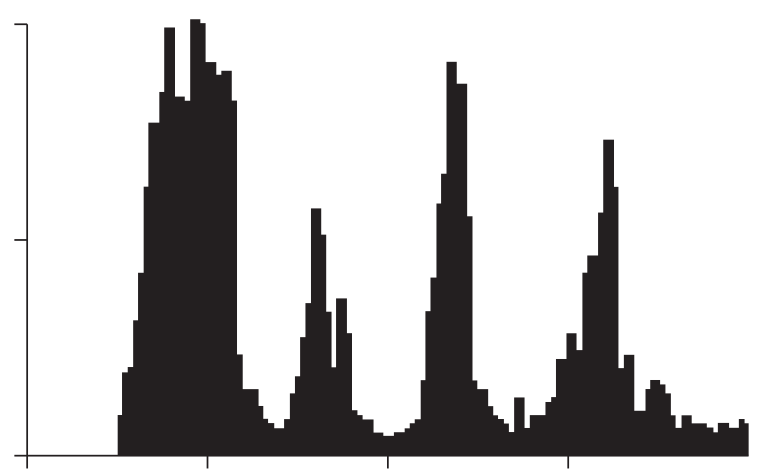

f)

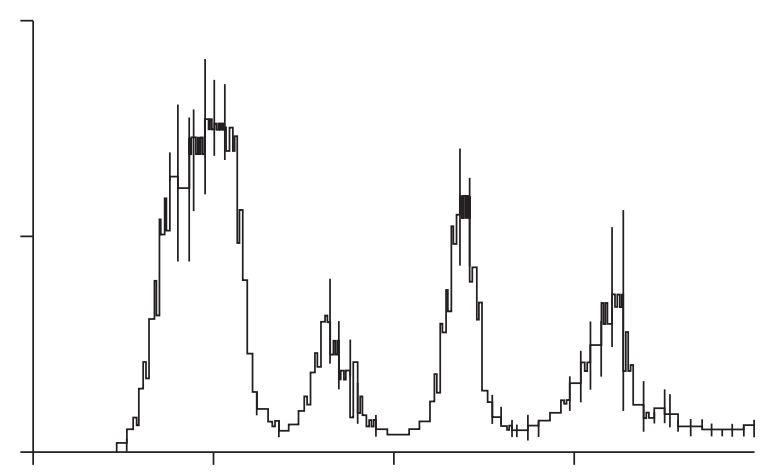

h)

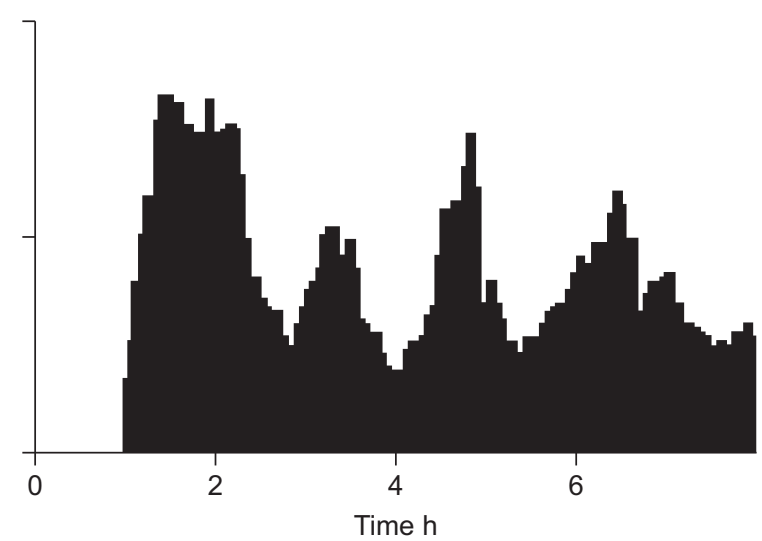

FIGURE 2. Example of child sleep analysis a, c, e, g) before adenotonsillectomy (AT) (apnoea-hypopnoea index (AHI) 27.5 events $\cdot h^{-1}$ ) and b, d, f, h) 3.5 months after AT (AHI 0.3 events $\left.\cdot h^{-1}\right)$. a, b) Visually scored sleep stages; c, d) time course of $\delta$ energy, i.e. slow-wave activity (SWA) (0.75-4 Hz; derivation C3/A2); e, f) smoothed $\delta$ energy (12 epochs moving average); g, h) slope of slow waves, i.e. the ratio of the most negative peak and the time to next zero crossing. SWA and sleep stages were plotted for 30-s epochs. W: waking; REM: rapid eye movement; S1: sleep stage 1; S2: sleep stage 2; S3+4: sleep stages 3 and 4, i.e. slow-wave sleep. 

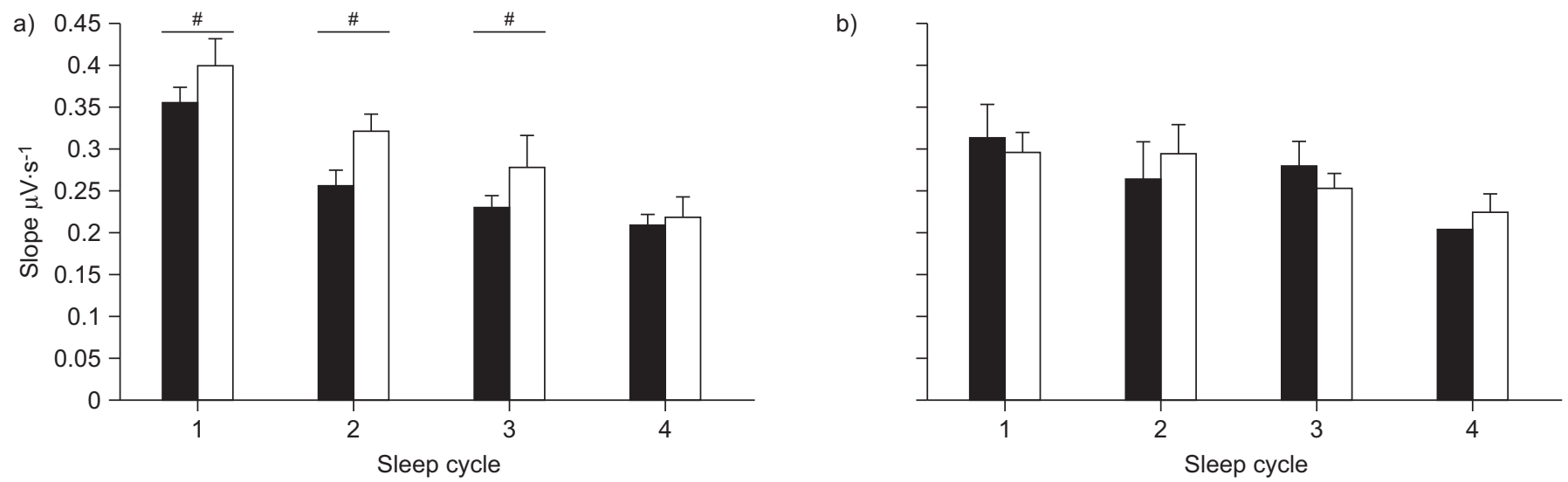

FIGURE 3. Time course of slow-wave slopes in the a) treated and b) comparison groups. Data are presented as mean \pm SE normalised slope of slow waves for consecutive non-rapid eye movement sleep (NREM) cycles. On repeated polysomnography studies, there was significant improvement of slow-wave slope following adenotonsillectomy across first three NREM sleep cycles.

- : before; $\square$ : after. ${ }^{*}$ : treatment factor $F=4.7, p<0.03$.

were analysed [26]. In the current study, as in previous reports obtained from adults and children [15, 27, 28], treatment of sleep-disordered breathing improves slow wave sleep intensity. Thus, all of these studies suggest that sleep-disordered breathing acts upon decreased NREM sleep depth. Why this occurs is unclear. Adult OSA patients may terminate their apnoeas without clear EEG arousals, but may also stay awake longer [26]. The intensity of SWA correlates positively with the threshold to arouse human subjects or animals [12]. It is possible that in children with OSA, SWA may be forced into reduced sleep depth or other states, such as wakefulness, in order to maintain ventilation during sleep. The initial assumption was that children with OSA do not rouse from their respiratory events during sleep as often as adults do; therefore, sleep architecture is better preserved than in adults $[1,6]$. TAUMAN et al. [29] suggested that the decrease in the nonrespiratory fraction of arousals represents an effort to preserve sleep homeostasis in children with sleep-disordered breathing. It was suggested that as an effort to preserve sleep homeostasis, children with OSA will develop some form of neural habituation that attenuates the EEG response to upper airway obstruction [22].

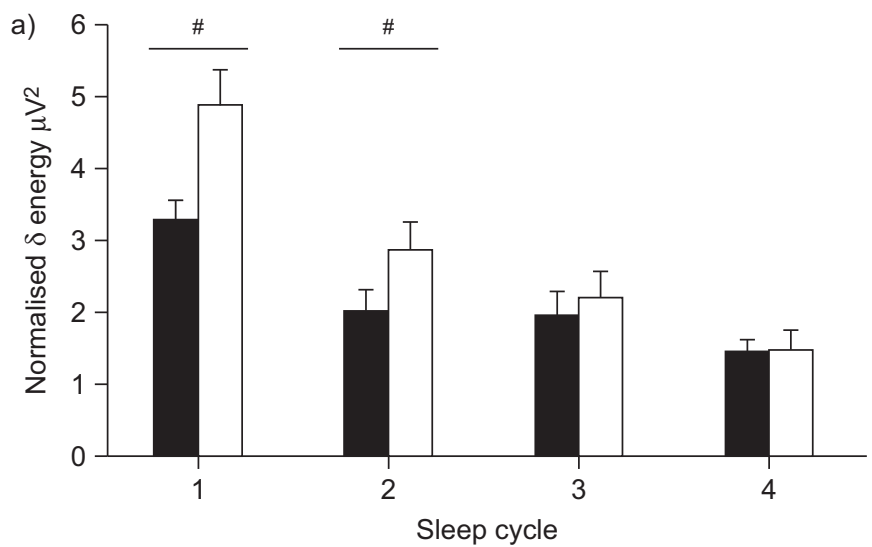

It is possible that the improvement in neurocognitive and behavioural function $[3,9,30]$, and growth hormone homeostasis $[2,31]$ following AT in children with sleep-disordered breathing are related to enhancement sleep intensity. A study using a computer model that simulates sleep homeostasis concluded that the decline of SWA could be predicted by a reduction of cortical synaptic strength, which is involved in the generation of slow wave sleep $[14,23]$. In adult OSA patients, it has been demonstrated that the excessive daytime sleepiness may be a result of lack of SWA during the first part of the night [15]. Moreover, experimentally induced sleep fragmentation can reduce SWA [32] and deplete hypothalamic growth hormone-releasing hormone, which is known to stimulate SWA and growth hormone secretion [33]. In support of this, OSA in children can lead to abnormal growth hormone homeostasis that significantly improves following AT [2, 31].

\section{Summary}

Children with untreated OSA exhibit decreased SWA level and slope during the night, indicating reduced sleep homeostasis. AT led to more physiological sleep homeostasis in children

b)

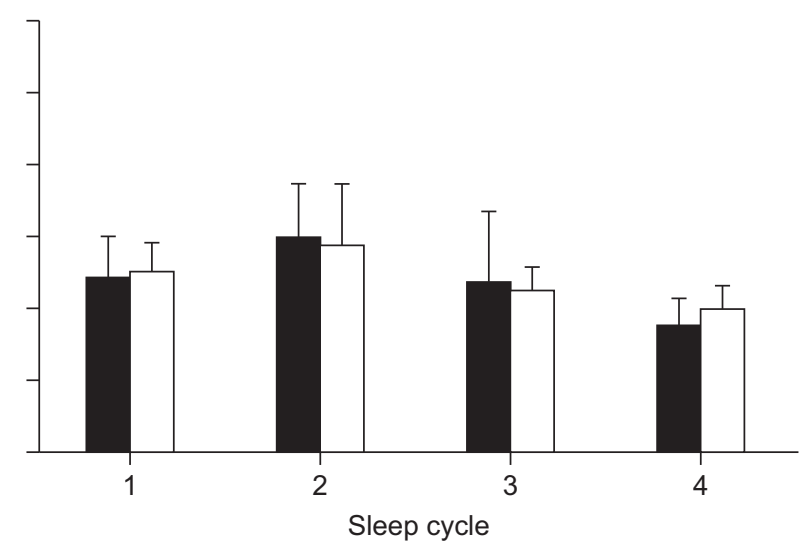

FIGURE 4. Time course of slow-wave activity (SWA) across four non-rapid eye movement (NREM) sleep cycles, for a) adenotonsillectomy (AT) and b) comparison-group children with obstructive sleep apnoea. Data are presented as mean \pm SE maximal normalised SWA for each NREM sleep cycle. AT significantly improved SWA in the first two sleep cycles. $\mathbf{\square}$ : before; $\square$ : after. ${ }^{\#}$ : treatment factor $\mathrm{F}=6.9, \mathrm{p}<0.01$. 
with OSA. SWA analysis may provide a useful addition to standard sleep-stage analyses in children with OSA.

\section{SUPPORT STATEMENT}

The study was supported by the Israel Science Foundation (award number 164/06).

\section{STATEMENT OF INTEREST}

None declared.

\section{REFERENCES}

1 Tal A, Bar A, Leiberman A, et al. Sleep characteristics following adenotonsillectomy in children with obstructive sleep apnea syndrome. Chest 2003; 124: 948-953.

2 Bar A, Tarasiuk A, Segev Y, et al. The effect of adenotonsillectomy on serum insulin-like growth factor-I and growth in children with obstructive sleep apnea syndrome. J Pediatr 1999; 135: 76-80.

3 Friedman BC, Hendeles-Amitai A, Kozminsky E, et al. Adenotonsillectomy improves neurocognitive function in children with obstructive sleep apnea syndrome. Sleep 2003; 26: 999-1005.

4 Marcus CL, Carroll JL, Koerner CB, et al. Determinants of growth in children with the obstructive sleep apnea syndrome. J Pediatr 1994; 125: 556-562.

5 Tarasiuk A, Simon T, Tal A, et al. Adenotonsillectomy in children with obstructive sleep apnea syndrome reduces health care utilization. Pediatrics 2004; 113: 351-356.

6 Goh DYT, Galster P, Marcus CL. Sleep architecture and respiratory disturbances in children with obstructive sleep apnea. Am J Resp Crit Care Med 2000; 162: 682-686.

7 Bhattacharjee R, Kheirandish-Gozal L, Spruyt K, et al. Adenotonsillectomy outcomes in treatment of OSA in children: a multicenter retrospective study. Am J Respir Crit Care Med 2010; 182: 676-683.

8 Tauman R, Gulliver TE, Krishna J, et al. Persistence of obstructive sleep apnea syndrome in children after adenotonsillectomy. J Pediatr 2006; 149: 803-808.

9 Montgomery-Downs HE, Crabtree VM, Gozal D. Cognition, sleep and respiration in at-risk children treated for obstructive sleep apnoea. Eur Respir J 2005; 25: 336-342.

10 Chervin RD, Fetterolf JL, Ruzicka DL, et al. Sleep stage dynamics differ between children with and without obstructive sleep apnea. Sleep 2009; 32: 1325-1332.

11 Borbely AA. A two-process model of sleep regulation. Hum Neurobiol 1982; 1: 195-204.

12 Tononi G. The neuro-biomolecular basis of alertness in sleep disorders. Sleep Med, 2005: Suppl. 1, S8-S12.

13 Huber R, Ghilardi MF, Massimini M, et al. Local sleep and learning. Nature 2004; 430: 78-81.

14 Esser SK, Hill SL, Tononi G. Sleep homeostasis and cortical synchronization: I. Modeling the effects of synaptic strength on sleep slow waves. Sleep 2007; 30: 1617-1630.

15 Heinzer R, Gaudreau H, Décary A, et al. Slow-wave activity in sleep apnea patients before and after continuous positive airway pressure treatment: contribution to daytime sleepiness. Chest 2001; 119: 1807-1813.

16 Brouillette RT, Hanson D, David R, et al. A diagnostic approach to suspected obstructive sleep apnea in children. J Pediatr 1984; 105: 10-14.

17 Rechtschaffen A, Kales A. A Manual of Standardized Terminology: Techniques and Scoring System for Sleep Stages of Human Subjects. Washington, US Public Health Service, 1968.

18 Feinberg I, Floyd TC. Systematic trends across the night in human sleep cycles. Psychophysiology 1979; 16: 283-291.

19 Jenni OG, Carskadon MA. Spectral analysis of the sleep electroencephalogram during adolescence. Sleep 2004; 27: 774-783.

20 Sleep Disorders Atlas Task Force. EEG arousals: scoring rules and examples. Sleep 1992; 15: 173-184.

21 American Thoracic Society: Standards and indications for cardiopulmonary sleep studies in children. The official statement of the American Thoracic Society. Am J Respir Crit Care Med 1996; 153: 866-878.

22 Bandla HP, Gozal D. Dynamic changes in EEG spectra during obstructive apnea in children. Pediatr Pulmonol 2000; 29: 359-365.

23 Riedner BA, Vyazovskiy VV, Huber R, et al. I. Sleep homeostasis and cortical synchronization: III. A high-density EEG study of sleep slow waves in humans. Sleep 2007; 30: 1643-1657.

24 Campbell IG, Feinberg I. Longitudinal trajectories of non-rapid eye movement $\delta$ and $\theta$ EEG as indicators of adolescent brain maturation. Proc Natl Acad Sci USA 2009; 106: 5177-5180.

25 Reuveni H, Chapnick G, Tal A, et al. Sleep fragmentation in children with Atopic Dermatitis. Arch Pediatr Adolesc Med 1999; 153: 249-253.

26 Guilleminault C, Do Kim Y, Chowdhuri S, et al. Sleep and daytime sleepiness in upper airway resistance syndrome compared to obstructive sleep apnoea syndrome. Eur Respir J 2001; 17: 838-847.

27 Guilleminault C, Lee JH, Chan A, et al. Non-REM-sleep instability in recurrent sleepwalking in pre-pubertal children. Sleep Med 2005; 6: 515-521.

28 Guilleminault C, Kirisoglu C, da Rosa AC, et al. Sleepwalking, a disorder of NREM sleep instability. Sleep Med 2006; 7: 163-170.

29 Tauman R, O'Brien LM, Holbrook CR, et al. Sleep pressure score: a new index of sleep disruption in snoring children. Sleep 2004; 27: 274-278.

30 O'Brien LM, Holbrook CR, Mervis CB, et al. Sleep and neurobehavioral characteristics of 5- to 7-year-old children with parentally reported symptoms of attention deficit/hyperactivity disorder. Pediatrics 2003; 111: 554-563.

31 Bonuck KA, Freeman K, Henderson. Growth and growth biomarker changes after adenotonsillectomy: systematic review and meta-analysis. J Arch Dis Child 2009; 94: 83-91.

32 Landsness EC, Crupi D, Hulse BK, et al. Sleep-dependent improvement in visuomotor learning: a causal role for slow waves. Sleep 2009; 32: 1273-1284.

33 Obal F Jr, Krueger JM. Biochemical regulation of non-rapid-eyemovement sleep. Front Biosci 2003; 8: d520-d550. 